\title{
Mathematical formalism of an internal air flow through a vortex tube machine of Hilsch-Ranque type: An analytical representation for velocity field
}

\author{
J. Venetis ${ }^{1}$ and E. Sideridis ${ }^{2,3 *}$ \\ ${ }^{1}$ School of Civil Engineering National Technical University (NTU) Athens, Greece. \\ ${ }^{2}$ School of Applied Mathematics and Physical Sciences National Technical University (NTU) Athens, Greece. \\ ${ }^{3}$ Section of Mechanics, 5 Heroes of Polytechnion Avenue GR - 15773 Athens, Greece.
}

Accepted 6 March, 2013

\begin{abstract}
In this paper, we will attempt to derive an analytical representation of velocity field for the concept of an internal air flow inside a vortex tube machine of Hilsch-Ranque type. The basic assumption here is that the flux field is assumed to be steady and incompressible. The vector which denotes instantaneous velocity, for the description of the flux field via Lagrangian stand point, will be expressed in a cylindrical coordinate system with orthonormal basis in the center line of the cross section of this vortex tube. We also assume here, that the aforementioned flux field varies only in radial direction, hence velocity vector is actually an one-dimensional damped circular helix trajectory with variable pitch.
\end{abstract}

Key words: Vortex tube, homographic function, vortex lines, helix trajectory.

\section{INTRODUCTION}

This vortex tube machine was firstly invented in 1931 by a Frenchman Physicist named Georges J. Ranque when he was studying processes in a dust separated cyclone (Fulton, 1950; Reynolds, 1962). The patent and idea was abandoned for several years until 1947, when a German engineer Rudolf Hilsch modified the design of the tube (De Vera, 2010; Eiamsa-ard and Promvonge, 2007). Particularly, R. Hilsch improved the initial design of this device by constructing a number of tubes and published experimental data with respect to their operation (Guillaume and Jolly, 2001). Since then, many researchers have tried to find ways to optimize its efficiency.

The vortex tube is actually a mechanical device that separates a compressed gas into hot and cold streams. It has no moving parts (Hilsch, 1947). Pressurized gas is injected tangentially into a swirl chamber and accelerated to a high rate of rotation. Due to the conical nozzle at the end of the tube, only the outer shell of the compressed gas is allowed to escape at that end. The remainder of the gas is forced to return in an inner vortex of reduced diameter within the outer vortex (Gutsol, 1997; Hilsch, 1947). There are different explanations for the effect and there is debate on which explanation is best or correct.

What is usually agreed upon is that the air in the tube experiences mostly "solid body rotation" which means that angular velocity of the inner gas is the same as that of the outer gas. This is different from what most consider standard vortex behavior - where inner fluid spins at a higher rate than outer fluid. The behavior of "solid body rotation" is probably due to the long length of time during which each parcel of air remains in the vortex allowing friction between the inner parcels and outer 
parcels to have a notable effect (Sibulkin, 1961). Besides during the vortex stretching procedure the angular momentum of the fluid matter stays invariant.

This vortex tube machine was firstly invented in 1931 by a Frenchman Physicist named Georges J. Ranque when he was studying processes in a dust separated cyclone (Fulton, 1950; Reynolds, 1962). The patent and idea was abandoned for several years until 1947, when a German engineer Rudolf Hilsch modified the design of the tube (De Vera, 2010; Eiamsa-ard and Promvonge, 2007). Particularly, R. Hilsch improved the initial design of this device by constructing a number of tubes and published experimental data with respect to their operation (Guillaume and Jolly, 2001). Since then, many researchers have tried to find ways to optimize its efficiency.

The vortex tube is actually a mechanical device that separates a compressed gas into hot and cold streams. It has no moving parts (Hilsch, 1947). Pressurized gas is injected tangentially into a swirl chamber and accelerated to a high rate of rotation. Due to the conical nozzle at the end of the tube, only the outer shell of the compressed gas is allowed to escape at that end. The remainder of the gas is forced to return in an inner vortex of reduced diameter within the outer vortex (Gutsol, 1997; Hilsch, 1947). There are different explanations for the effect and there is debate on which explanation is best or correct.

What is usually agreed upon is that the air in the tube experiences mostly "solid body rotation" which means that angular velocity of the inner gas is the same as that of the outer gas. This is different from what most consider standard vortex behavior - where inner fluid spins at a higher rate than outer fluid. The behavior of "solid body rotation" is probably due to the long length of time during which each parcel of air remains in the vortex allowing friction between the inner parcels and outer parcels to have a notable effect (Sibulkin, 1961). Besides during the vortex stretching procedure the angular momentum of the fluid matter stays invariant.

The chief characteristic of this peculiar device is separation of the introduced air into hot and cold fractions. His interest lay in its potentialities as a refrigerating unit, but apparently he was unable to develop it satisfactorily (Eckert and Hartnett, 1955; Lewellen, 1962; Linderstrom-Lang, 1967). His work attracted rather widespread interest and, as a result, a number of relevant publications have appeared.

Some further investigations by Physicists and Engineers have been actually of particular significance, because they reported measurable mass separation gas mixtures and discussed theories of operation (Ahlborn and Gordon, 2000; De Vera, 2010; Piralishili et al., 2005). However, no explanations have been attempted. Most of other reports have mainly dealt with the application of the tube to refrigeration (Ahlborn et al., 1996; Gao et al., 2005; Eckert and Hartnett, 1955; Khodorkov et al., 2003).
Until today, there is no single theory that explains the radial temperature separation. However, the operating cost of this machine is generally low and its applications are indeed various (Tassios, 2006).

The cross sectional area with a plane parallel to axis $\mathrm{zz}^{\prime}$ of this aforementioned hook-up is represented in Figure 1.

As concerns the instantaneous velocity distribution, the following relationship holds:

$\vec{V}=f(r) \cdot \vec{e}_{\theta}$

where the quantity $f(r): R \rightarrow R$ denotes a real singlevalued continuous function

\section{PRESENTATION OF THE MATHEMATICAL FORMALISM}

According to elementary Analytical Geometry, the transform relations from cylindrical to Cartesian coordinates hold:

$\vec{\imath}=\vec{e}_{r} \cdot \cos \theta-\vec{e}_{\theta} \cdot \sin \theta$

$\vec{j}=\vec{e}_{r} \cdot \sin \theta+\vec{e}_{\theta} \cdot \cos \theta$

$\vec{k}=\vec{e}_{z}$

where $\vec{e}_{\theta}$ denotes the unit vector being collinear to the component: $\overrightarrow{\mathrm{V}_{\theta}}$ at a Cylindrical Coordinate System and in fact is able to be written out equivalently with respect to its own projections at the Cartesian Coordinate System Oxyz, as follows:

$\vec{e}_{\theta}=-\sin \theta \vec{\imath}+\cos \theta \vec{j}+0 \vec{k}$

Moreover, the unit vector which is collinear to the radial component: $\vec{V}_{r}$ can be also written out as

$\vec{e}_{r}=\cos \theta \vec{\imath}+\sin \theta \vec{j}+0 \vec{k}$

Hence, it follows:

$\left[\begin{array}{l}V_{r} \\ V_{\theta} \\ V_{z}\end{array}\right]=\left[\begin{array}{ccc}\cos \theta & \sin \theta & 0 \\ -\sin \theta & \cos \theta & 0 \\ 0 & 0 & 1\end{array}\right] \cdot\left[\begin{array}{c}V_{x} \\ V_{y} \\ V_{z}\end{array}\right] \Leftrightarrow$

$\left[\begin{array}{l}V_{x} \\ V_{y} \\ V_{z}\end{array}\right]=\left[\begin{array}{ccc}\cos \theta & \sin \theta & 0 \\ -\sin \theta & \cos \theta & 0 \\ 0 & 0 & 1\end{array}\right]^{-1} \cdot\left[\begin{array}{l}V_{r} \\ V_{\theta} \\ V_{z}\end{array}\right] \Leftrightarrow$ 


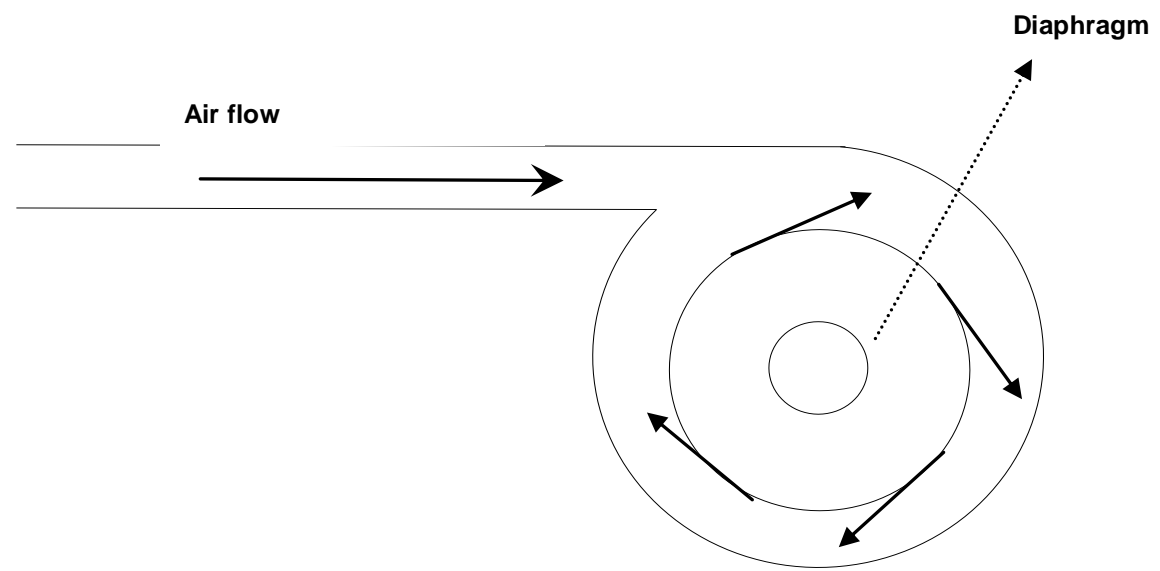

Figure 1. Cross-section with a plane parallel to axis zz.'

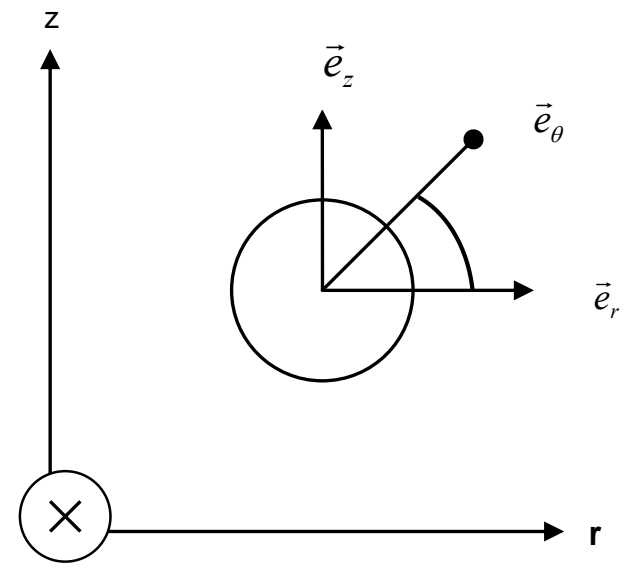

Figure 2. Motionless local cylindrical system customized at the "eye» of the maximum vortex

$$
\begin{aligned}
& V_{x}=V_{r} \cdot \cos \theta-V_{\theta} \cdot \sin \theta \\
& V_{y}=V_{r} \cdot \sin \theta+V_{\theta} \cdot \cos \theta \\
& V_{z}=V_{z}
\end{aligned}
$$

Since the flux field that we examine is primarily assumed to vary only in the radial direction, it implies that velocity component $V_{\theta}$ is identically zero (Figure 2).

Nevertheless, the generic transform relations read

$$
\begin{aligned}
& r=\left(x^{2}+y^{2}\right)^{0.5} \\
& \theta=\arctan \left(\frac{y}{x}\right) \forall x>0 \wedge y \geq 0
\end{aligned}
$$

$$
z=z
$$

Hence, the one-dimensional instantaneous velocity at the vortex tube emerges via the following representation:

$$
\begin{aligned}
& \vec{V}=f(r) \cdot \vec{e}_{\theta}=f(r) \cdot((-\sin (\theta+\omega t) \vec{\imath}+(\cos (\theta+\omega t) \vec{j}) \Leftrightarrow \\
& \vec{V}=f(r) \cdot((-\sin (\theta+\omega t) \vec{\imath}+(\cos (\theta+\omega t) \vec{j}+0 \vec{k})
\end{aligned}
$$

In this paper, we will make a specific implementation for a characteristic rational function, particularly for the homographic one, that is

$$
f(r)=\frac{\alpha \cdot r+\beta}{\gamma \cdot r+\delta}
$$

where: $r \neq-\frac{\delta}{\gamma}$

It is also known from elementary Calculus, that Equation (6) can be exhibited equivalently in the following form:

$$
f(r)=\frac{\alpha \cdot r+\beta}{\gamma \cdot r+\delta} \Leftrightarrow
$$

$$
f(r)=\frac{\alpha}{\gamma}+\frac{\frac{\beta \gamma-\alpha \delta}{\gamma^{2}}}{r+\frac{\delta}{\gamma}}=k+\frac{\lambda}{r+\mu}
$$

where $\lambda, \mu \in R$

So we can deduce: 


$$
\begin{aligned}
& \frac{d f(r)}{d r}=\frac{a(\gamma \cdot r+\delta)-\gamma \cdot(\alpha \cdot r+\beta)}{(\gamma \cdot r+\delta)^{2}} \Leftrightarrow \\
& \frac{d f(r)}{d r}=-\frac{(\beta \gamma-\alpha \delta)}{(\gamma \cdot r+\delta)^{2}}=-\frac{\lambda}{(\gamma \cdot r+\delta)^{2}}
\end{aligned}
$$$$
V_{x}=\left(k+\frac{\lambda}{r+\mu}\right) \cdot(-\sin (\theta+\omega t))
$$

$$
V_{y}=\left(k+\frac{\lambda}{r+\mu}\right) \cdot(\cos (\theta+\omega t))
$$

If we keep track of the equation above, we can pinpoint that if $\lambda \neq 0$, then the range of the function: $f(r)$ does not occur any local extrema.

$V_{z}=0$

Thus, we can figure out

Thus, the instantaneous velocity at the vortex tube, in Cartesian coordinates can be recaptured via the following representation:

$$
\left[\begin{array}{l}
V_{r} \\
V_{\theta} \\
V_{z}
\end{array}\right]=\left[\begin{array}{ccc}
\cos \theta & \sin \theta & 0 \\
-\sin \theta & \cos \theta & 0 \\
0 & 0 & 1
\end{array}\right] \cdot\left[\begin{array}{c}
V_{x} \\
V_{y} \\
V_{z}
\end{array}\right] \Rightarrow
$$

$$
\left[\begin{array}{l}
V_{r} \\
V_{\theta} \\
V_{z}
\end{array}\right]=\left[\begin{array}{c}
\cos \theta\left(k+\frac{\lambda}{r+\mu}\right) \cdot(-\sin (\theta+\omega t))+\sin \theta\left(k+\frac{\lambda}{r+\mu}\right) \cdot(\cos (\theta+\omega t)) \\
-\sin \theta\left(k+\frac{\lambda}{r+\mu}\right) \cdot(\cos (\theta+\omega t))+\cos \theta\left(k+\frac{\lambda}{r+\mu}\right) \cdot(\cos (\theta+\omega t)) \\
V_{z}
\end{array}\right]
$$

The corresponding boundary conditions for the particular original problem are:

$$
\begin{aligned}
& r \rightarrow 0 \Rightarrow V_{y} \rightarrow 0 \Rightarrow \\
& \theta+\omega t=2 n \pi \\
& r \rightarrow 0 \Rightarrow V_{x}=\max \Rightarrow \\
& \max V_{y}=k+\frac{\lambda}{\mu} \\
& r=\max \Rightarrow V_{x}=0 \\
& r=\max \Rightarrow V_{y}=0
\end{aligned}
$$

Concurrently, the position vector components in Cartesian Ccoordinates are:

$x(t)=r \cos (\theta+\omega t)$ $y(t)=r \sin (\theta+\omega t)$

$$
z(t)=z(t)
$$

where: $z(t): R \rightarrow R$ constitutes an arbitrary single - valued function.

In the sequel, according to Lagrangian formalism we deduce:

$$
\begin{gathered}
V_{x}=\frac{d x(t)}{d t} \\
\wedge \\
V_{y}=\frac{d y(t)}{d t}
\end{gathered}
$$

Hence, with the latter two equations in hand in accordance with the set of Equation (9) we can eventually infer

$V_{x}=\left(k+\frac{\lambda}{\left(x^{2}+y^{2}\right)^{0.5}+\mu}\right) \cdot\left(-\frac{y}{\left(x^{2}+y^{2}\right)^{0.5}}\right)$ 
$V_{y}=\left(k+\frac{\lambda}{\left(x^{2}+y^{2}\right)^{0.5}+\mu}\right) \cdot\left(\frac{x}{\left(x^{2}+y^{2}\right)^{0.5}}\right)$

\section{DESCRIPTION OF THE STREAM AND VORTEX LINES}

It is known from elementary Vector Calculus that the following invariant vector identity holds true, for an infinitesimal part of an arbitrary stream line $d \vec{s}$ :

$$
\begin{aligned}
& \vec{V} \times d \vec{s}=\overrightarrow{0} \Rightarrow \\
& d x=d r \cos \theta-r \sin \theta d \theta \\
& d y=d r \sin \theta-r \cos \theta d \theta \\
& d z=d z
\end{aligned}
$$

Hence, we can result to the following statement

$$
\frac{d r}{V_{r}}=\frac{r d \theta}{V_{\theta}}=\frac{d z}{V_{z}}
$$

Besides, the vortex lines of any flow pattern are defined by the following set of equations

$$
\frac{d x}{\frac{\partial V_{z}}{\partial y}-\frac{\partial V_{y}}{\partial z}}=\frac{d y}{\frac{\partial V_{x}}{\partial z}-\frac{\partial V_{z}}{\partial x}}=\frac{d z}{\frac{\partial V_{x}}{\partial y}-\frac{\partial V_{y}}{\partial x}}
$$

Thus, according to the definition of the angular velocity we infer

$$
\frac{d x}{\omega_{x}}=\frac{d y}{\omega_{y}}=\frac{d z}{\omega_{z}}=\frac{d(x+y+z)}{\omega_{x}+\omega_{y}+\omega_{z}}
$$

In continuing, according to the definition of the curl of a vector function in cylindrical coordinates we can also deduce

$$
\left[\begin{array}{c}
\omega_{r} \\
\omega_{\theta} \\
\omega_{z}
\end{array}\right]=\frac{1}{2 r}\left[\begin{array}{ccc}
\vec{e}_{r} & r \vec{e}_{\theta} & \vec{e}_{z} \\
\partial / \partial r & \partial / \partial \theta & \partial / \partial z \\
V_{r} & r V_{\theta} & V_{z}
\end{array}\right]
$$

Next, by considering a right-hand circular helix of pitch equal to: $2 b \pi$ the drawn from the origin position vector $\vec{r}(x(t), y(t), z(t))$ is given by:

$\vec{r}(t)=a \cos \omega t \vec{i}+a \sin \omega t \vec{j}+b t \vec{k}$ where $a, b, \omega>0$

The corresponding arc length being traced by an arbitrary trip of position vector is given by the integral:

$s=\int_{0}^{t}\left|r^{\prime}(\tau)\right| d \tau=t \cdot \sqrt{a^{2} \omega^{2}+b^{2}}$

It is also known from Differential Geometry that all curves which have both the same curvature function and same torsion function are congruent.

Hence the following conjunction holds

$$
\begin{aligned}
& k(s)=\frac{a \omega^{2}}{a^{2} \omega^{2}+b^{2}} \\
& \wedge \\
& \tau(s)=\frac{b \omega}{a^{2} \omega^{2}+b^{2}}
\end{aligned}
$$

On the other hand, according to Lagrangian formalism for the fluid motion along an arbitrary stream line of the flux field that we study the following equality holds:

$$
\vec{V}=\frac{d \vec{r}(t)}{d t}=\frac{d s(t)}{d t} \cdot \vec{e}_{t}
$$

By operating the material derivatives at both members of Equation (22) we obtain

$\frac{\partial \vec{V}}{\partial t}+\vec{V} \cdot \operatorname{grad} \vec{V}=\frac{d^{2} s(t)}{d t^{2}} \cdot \vec{e}_{t}+\frac{d s(t)}{d t} \cdot \frac{d \vec{e}_{t}}{d t}$

We emphasize that the above relationship holds along any stream line of the investigated flux field.

Besides, the following implication also holds:

$\left|\vec{e}_{t}\right|=1 \Rightarrow$

$\left|\vec{e}_{t}\right|^{2}=1 \Rightarrow$

$\vec{e}_{t} \cdot \vec{e}_{t}=1 \Rightarrow$

$$
\begin{aligned}
& \frac{d\left(\vec{e}_{t} \cdot \vec{e}_{t}\right)}{d t}=0 \Rightarrow \\
& 2 \frac{d \vec{e}_{t}}{d t} \cdot \vec{e}_{t}=0 \Rightarrow
\end{aligned}
$$

$\frac{d \vec{e}_{t}}{d t}=0$

$\vee$

$\frac{d \vec{e}_{t}}{d t} \perp \vec{e}_{t}$ 
In the sequel, if we consider the unit vector $\vec{e}_{n}$ which is collinear to the vector $\frac{d \vec{e}_{t}}{d t}$ and also outward vertical to the aforementioned surface, $\left(\vec{e}_{n} \quad\right.$ is referred to as principal normal vector), it implies that:

$$
\begin{aligned}
& \frac{\mathrm{d} \overrightarrow{\mathrm{r}}}{d t}=\frac{\mathrm{d} \overrightarrow{\mathrm{r}}}{\mathrm{ds}(\mathrm{t})} \cdot \frac{\mathrm{ds}(\mathrm{t})}{d t} \stackrel{\text { eqn.(22) }}{\Rightarrow} \\
& \frac{d s(t)}{d t} \cdot \vec{e}_{t}=\frac{d \vec{r}}{d s(t)} \cdot \frac{d s(t)}{d t} \Rightarrow \\
& \vec{e}_{t}=\frac{d \vec{r}}{d s(t)} \Rightarrow \\
& \frac{1}{\vec{e}_{t}}=\frac{d s(t)}{d \vec{r}}
\end{aligned}
$$

Next, by differentiating both members of the latter equality with respect to variable $s$ we can figure out

$$
\begin{aligned}
& -\frac{\frac{d \vec{e}_{t}}{d s}}{\left(\vec{e}_{t}\right)^{2}}=\frac{d}{d s}\left(\frac{d \vec{r}}{d s(t)}\right) \Rightarrow \\
& \frac{d \vec{e}_{t}}{d s}=-\frac{d}{d s}\left(\frac{d \vec{r}}{d s(t)}\right) \cdot \vec{e}_{t}^{2} \Rightarrow \\
& \frac{d \vec{e}_{t}}{d s}=-\frac{d}{d s}\left(\frac{d \vec{r}}{d s(t)}\right)
\end{aligned}
$$

Moreover, it is known from Differential Geometry that the following relationship holds:

$$
\vec{e}_{n}=\frac{1}{k(s)} \cdot \frac{d}{d s}\left(\frac{d \vec{r}}{d s(t)}\right)=R(s) \frac{d^{2} \vec{r}}{d s^{2}}
$$

Hence, we can eventually result to the following statement:

$$
\begin{aligned}
& \frac{d \vec{e}_{t}}{d s}=-\frac{1}{R(s)} \cdot \vec{e}_{n} \Rightarrow \\
& \frac{d \vec{e}_{t}}{d t}=-\frac{1}{R(s)} \cdot \frac{d s}{d t} \cdot \vec{e}_{n}
\end{aligned}
$$

Thus, Equation (23) can be equivalently represented in the following rephrased form

$$
\frac{\partial \vec{V}}{\partial t}+\vec{V} \cdot \operatorname{grad} \vec{V}=\frac{d^{2} s(t)}{d t^{2}} \cdot \vec{e}_{t}-\frac{1}{R} \cdot\left(\frac{d s(t)}{d t}\right)^{2} \cdot \vec{e}_{n}
$$

Alongside, from Vector Calculus the following identity holds:

$$
\frac{\partial \vec{V}}{\partial t}+\vec{V} \cdot \operatorname{grad} \vec{V} \equiv \frac{\partial \vec{V}}{\partial t}+\operatorname{grad}\left(\frac{V^{2}}{2}\right)-\vec{V} \times(\operatorname{grad} \times \vec{V})
$$

where $V$ denotes the algebraic rate of vector $\vec{V}$ regardless of its circumstantial orientation.

Hence, by considering an element of length $d \vec{s}$ along an arbitrary stream line and operating the dot product in Equation (28) it implies:

$$
\begin{aligned}
& \frac{\partial \vec{V}}{\partial t} \cdot d \vec{s}+\operatorname{grad}\left(\frac{V^{2}}{2}\right) \cdot d \vec{s}=\frac{d^{2} s(t)}{d t^{2}} \cdot \vec{e}_{t} \cdot d \vec{s}-\frac{1}{R} \cdot\left(\frac{d s(t)}{d t}\right)^{2} \cdot \vec{e}_{n} \cdot d \vec{s} \\
& \vec{e}_{n} \perp d \vec{s} \\
& \quad \Leftrightarrow
\end{aligned}
$$$$
\frac{\partial \vec{V}}{\partial t} \cdot d \vec{s}+\operatorname{grad}\left(\frac{V^{2}}{2}\right) \cdot d \vec{s}=\frac{d^{2} s(t)}{d t^{2}} \cdot \vec{e}_{t} \cdot d \vec{s}
$$

The arc length whose endpoints define this orientated segment is traced by a trip of position vector $\vec{r}(t)$ and is given by the integral:

$$
s=\int_{0}^{t}\left|r^{\prime}(\tau)\right| d \tau
$$

We also observe that Equation (30) has been derived without the distinction between inviscid and viscous flow patterns and holds for stream lines network for both states of flow.

Furthermore, since $\vec{e}_{t} \cdot d \vec{s}=\left|\vec{e}_{t}\right| \cdot|d \vec{s}|=|d \vec{s}|$, Equation (30) yields

$$
\frac{\partial \vec{V}}{\partial t}+\operatorname{grad}\left(\frac{V^{2}}{2}\right)=\frac{d^{2} s(t)}{d t^{2}} \cdot\left(\frac{d \vec{s}}{|d \vec{s}|}\right)^{-1}
$$

where the unit vector: $\frac{d \vec{s}}{|d \vec{s}|}$ varies only in direction, denoting indeed the orientation of each arc whose length gets rates from the range of the scalar function: $s(t)$.

Apparently, Equation (32) holds for any stream lines network of incompressible flow patterns (internal and 
wake ones, as well as free turbulence).

Apart from these, according to Lagrangian formalism for a fluid moving particle in a cylindrical coordinate system the following formulas for instantaneous velocity and acceleration also hold true:

$$
\vec{V}=\frac{d \vec{r}}{d t}=\frac{d r}{d t} \vec{e}_{r}+r \frac{d \theta}{d t} \vec{e}_{\theta}
$$

The two above vector terms denote radial and transverse velocity components.

$$
\vec{a}=\left[\frac{d^{2} r}{d r^{2}}-r\left(\frac{d \theta}{d t}\right)^{2}\right] \vec{e}_{r}+\left[\frac{1}{r} \frac{d}{d t}\left(r^{2} \frac{d \theta}{d t}\right)\right] \vec{e}_{\theta}
$$

(The two above vector terms denote radial and transverse acceleration components).

Equations (34) and (28) are indeed identically equivalent to one another as the interface of two phenomenological points of view for the inceptive original problem, (that is, the approach of a bundle of moving particles and the approach of a continuum medium) and must be assessed in association with Equation (17).

Since we have focused on steady flux fields, by considering the geometry of the vortex tube as a succession of its cross sectional areas with planes parallel to axis $z z^{\prime}$ we can neglect the vorticity transfer in space and therefore the initial original problem substantially reduces to a superposition of twodimensional flux fields.

In two-dimensional flux fields the unit vector $\frac{d \vec{s}}{|d \vec{s}|}$ can be represented as follows:

Let us consider the generic form of a line in polar coordinates, defined by a point: $\left(r_{1}, \theta_{1}\right)$ on a stream line and another one: $\left(r_{2}, \theta_{2}\right)$ which is localized on the tangent line which passes from the point $\left(r_{1}, \theta_{1}\right)$, that is,

$r_{1} \cdot r \sin \left(\theta-\theta_{1}\right)+r_{2} \cdot r \sin \left(\theta-\theta_{2}\right)=r_{1} \cdot r_{2} \sin \left(\theta_{2}-\theta_{1}\right)$

Then, the specific line which passes through the pole: $(0,0)$ as well as the point: $\left(r_{1}, \theta_{1}\right)$ has the form:

$r_{1} \cdot r \sin \left(\theta-\theta_{1}\right)=0$

Besides the corresponding tangent line on $\left(r_{1}, \theta_{1}\right)$ which obviously is normal to the above line, has also the form: $r \cos \left(\theta-\theta_{1}\right)=r_{1}$

This latter equation, must be verified by the coordinates of the point $\left(r_{2}, \theta_{2}\right)$

Then the magnitude $|d \vec{s}|$ is estimated as follows:

$$
|d \vec{s}|=\sqrt{r_{1}^{2}+r_{2}^{2}-2 r_{1} r_{2} \cos \left(\theta_{2}-\theta_{1}\right)}
$$

where the following equality holds:

$$
\cos \left(\theta_{2}-\theta_{1}\right)=\frac{r_{1}}{r_{2}}
$$

Then it follows:

$$
\begin{aligned}
& |d \vec{s}|=\sqrt{r_{1}^{2}+r_{2}{ }^{2}-2 r_{1}^{2}} \Leftrightarrow \\
& |d \vec{s}|=\sqrt{r_{2}^{2}-r_{1}^{2}}=\sqrt{r_{2}-r_{1}} \cdot \sqrt{r_{2}+r_{1}}
\end{aligned}
$$

Hence, we eventually obtain:

$$
\frac{d \vec{s}}{|d \vec{s}|}=\frac{\left(r_{2}-r_{1}, \theta_{2}-\theta_{1}\right)}{\sqrt{r_{2}-r_{1}} \cdot \sqrt{r_{2}+r_{1}}}=\left(\frac{\sqrt{r_{2}-r_{1}}}{\sqrt{r_{2}+r_{1}}}, \frac{\theta_{2}-\theta_{1}}{\sqrt{r_{2}-r_{1}} \cdot \sqrt{r_{2}+r_{1}}}\right)
$$

Apparently, this latter equation holds along any crosssection of the cylindrical solid boundary as well, since it consists in marginally a stream surface.

\section{DISCUSSION}

This paper was intended to derive an analytical representation of velocity field for the concept of an internal viscous air flow inside a vortex tube machine of Hilsch-Ranque type.

The basic assumption here is that the flux field is assumed to be steady and incompressible throughout. However, the compressibility of gases can be neglected if and only if the flow velocity is small as compared to the local acoustic velocity (Lions 1996, 1998). This actually implies that either the ratio $\frac{\Delta \rho}{\rho}$ is considerably smaller than unity, or equivalently $\frac{\mathrm{Ma}^{2}}{2}<<1$. Therefore, all the mathematical results that occur on this work hold true only on the range of validity of the above two equivalent statements. 
We have also supposed, that the motivated flux field varies only in radial direction, hence velocity vector is actually one-dimensional vanished circular helix trajectory with obviously decreased pitch.

This approach actually implies that the three dimensional vorticity transfers have been primarily neglected.

On the other hand by means of Bernoulli equation in accordance with the presented mathematical formalism, one can also designate approximately the exact location where the static pressure changes sign along the tube as well as from wall to its centroid axis without neglecting the centrifugal effect (Arfken 1970; Farouk and Farouk, 2007).

In this case one can also take into account that if a conservative force shows no variation in one direction, then its component in that direction is the same everywhere (Arfken, 1970; Ladyzhenskaya, 1975). Therefore, with a proper choice of a Cartesian coordinate system the corresponding potential energy $E_{p}$ can be written out in the form:

$$
E_{p}=E_{p}(x, y)+\kappa z
$$

\section{where $\kappa$ is a constant number}

In the sequel, the above relationship can be easily modified in cylindrical coordinates. Nevertheless, the convergence of stream lines and the vortex lines in three dimensional internal flow patterns has several times significant changes from the simplified two dimensional flow field (Dafalias, 2003; Schlichting, 1979). Therefore, it could be investigated alternatively in the Euclidean or Cartesian space, by designing an isometric projection of the vortex tube machine and following CFD methods, since the geometry of this flow conductor is actually known from mathematical stand point.

We can also pinpoint that CFD methods can be very effective for the examination of this phenomenon since any cross sectional area of the interior boundary of this machine always constitutes indeed a positively orientated, not self-intersecting and continuously contracted curve.

\section{REFERENCES}

Arfken G (1970). Mathematical Methods for Physicists Second edition Academic Press New York.

Ahlborn B,Gordon $J$ (2000). The vortex tube as a classic thermodynamic refrigeration cycle. J. Appl. Phys. 88(6):3645-3653.

Ahlborn B, Camire J, Keller J (1996) Low-pressure vortex tubes. J. Phys. D: Appl. Phys. 29:1469-1472.

Dafalias I (2003). Continuum Mechanics, Lecture Notes for the Postgraduate Program at Applied Mechanics - NTUA, Athens.

De Vera G (2010). The Ranque-Hilsch Vortex Tube Mechanical Engineering--uc Berkeley.http://www.me.berkeley.edu/ gtdevera/ notes/vortextube.pdf.
Eckert ERG, Hartnett JP (1955). Experimental Study of the Velocity and Temperature Distribution in a High-Velocity Vortex-type Flow. Publication of Heat Transfer Laboratory, University of Minnesota, Minneapolis.

Eiamsa-ard S, Promvonge P (2007). Numerical investigation of the thermal separation in a Ranque-Hilsch vortex tube. Int. J. Heat Mass Transf. 50:821-832.

Farouk T, Farouk B, (2007). Large eddy simulations of the flow field and temperature separation in the Ranque-Hilsch vortex tube. Int. J. Heat Mass Transf. 50:4724-4735.

Fulton CD (1950). Comments on the vortex tube. J. ASRE Refrigerating Eng. 58:984.

Gao CM, Bosschaart KJ, Zeegers JCH, de Waele ATAM (2005). Experimental study on a simple Ranque-Hilsch vortex tube. Cryogenics, 45(3):173, 2005.

Guillaume D, Jolly J (2001). Demonstrating the achievement of lower temperatures with two-stage vortex tubes. Rev. Sci. Instrum. 72(8):3446-3448.

Gutsol AF (1997). The Ranque effect. Phys.-Usp. 40(6):639-658.

Hilsch R (1947). The use of the expansion of gases in a centrifugal field as cooling process. Rev. Sci. Instrum. 18(2):108-113.

Khodorkov IL, Poshernev NV, Zhidkov MA (2003). The vortex tube - A universal device for heating, cooling, cleaning and drying gases and separating gas mixtures. Chem. Pet. Eng. 39:7-8.

Ladyzhenskaya OA (1975). The mathematical theory of viscous incompressible flow Second English edition. Translated from the Russian by R.A. Silverman and J. Chu. Gordon and Breach Science Publishers New York.

Lewellen WS (1962). A solution for three-dimentional vortex flows with strong circulation. J. Fluid Mech. 14:420-432.

Linderstrom-Lang CU (1967.). On gas separation in Ranque-Hilsch vortex tubes. Z. Naturforschg. 22(a):835-837,112.

Lions PL (1996). Mathematical topics in fluid mechanics Incompressible models. Oxford Lecture Series in Mathematics and its Applications 3. Oxford University press, Volume 1.

Lions PL (1998). Mathematical topics in fluid mechanics - Compressible models. Oxford Lecture Series in Mathematics and its Applications 10. Oxford University press, Volume 2.

Piralishili, Sh A, Fuzeeva AA, Hydraulic MS (2005). Characteristics of Ranque-Hilsch energy separators. High Temp. 43(6):900-907.

Reynolds A (1962). A note on vortex-tube flow. J. Fluid Mech. 14:1820.

Schlichting H (1979). Boundary Layer Theory, $7^{\text {th }}$ Edition, Mc Graw Hill, New York.

Sibulkin M (1961). Unsteady, viscous, circular flow part 3. Application to the Ranque-Hilsch vortex tube. J. Fluid Mech. 12:269-293.

Stoker J (1969). Differential Geometry John Willey \& Sons.

Tassios D (2006). Applied Chemical Thermodynamics 2nd Edition NTUA Press, Athens. 